Communications in Physics, Vol.31, No. 1 (2021), pp. 23-33

DOI:10.15625/0868-3166/15178

\title{
INTERACTION BETWEEN TWO JACKIW-REBBI STATES IN INTERFACED BINARY WAVEGUIDE ARRAYS WITH CUBIC-QUINTIC NONLINEARITY
}

\author{
TRAN X. TRUONG ${ }^{1, \dagger}$, NGUYEN NHU XUAN $^{1}$, NGUYEN-THE QUANG $^{2}$, \\ NGUYEN VAN TOAN ${ }^{1}$ AND NGUYEN TUAN LINH ${ }^{1}$ \\ ${ }^{1}$ Department of Physics, Le Quy Don Technical University, \\ 236 Hoang Quoc Viet str., 10000 Hanoi, Vietnam \\ ${ }^{2}$ Department of Communications, Le Quy Don Technical University, \\ 236 Hoang Quoc Viet str., 100000 Hanoi, Vietnam \\ E-mail: ${ }^{\dagger}$ tranxtr@gmail.com
}

Received 25 June 2020

Accepted for publication 21 July 2020

Published 05 January 2021

\begin{abstract}
We study the coupling and switching effects of two discrete relativistic quantum JackiwRebbi states in interfaced binary waveguide arrays with cubic-quintic nonlinearity. Like in the case with Kerr nonlinearity, two Jackiw-Rebbi states can couple efficiently to each other in the low-power regime, show the switching effect in the intermediate-power regime, and possess the trapping effect in the high-power regime. However, in the case with cubic-quintic nonlinearity, if the input Jackiw-Rebbi state power is increased further, one can observe the quasi-linear coupling effect between two Jackiw-Rebbi states which has not been found between two Jackiw-Rebbi states in interfaced binary waveguide arrays with Kerr nonlinearity.
\end{abstract}

Keywords: soliton; binary waveguide array; cubic-quintic nonlinearity.

Classification numbers: 42.65.Tg; 42.81.Dp; 42.82.Et.

\section{INTRODUCTION}

Many interesting fundamental photonic phenomena such as discrete solitons (DSs) [1-3], discrete diffraction [1,4], diffractive resonant radiation [5] have been found in waveguide arrays (WAs). Waveguide arrays have also been used to simulate fundamental effects in nonrelativistic quantum mechanics such as Zener tunneling [6], and photonic Bloch oscillations [7]. Recently, binary waveguide arrays (BWAs) have been intensively used to investigate several fundamental 
relativistic quantum mechanics phenomena arising from the Dirac equation, e.g., Klein tunneling [8-10], Zitterbewegung [11], and Dirac solitons in the nonlinear regime [12-18].

In 2017, two localized states (one is the optical analogue of a special state which is well known as the Jackiw-Rebbi (JR) state emerging from the Dirac equation in the quantum field theory [19], and the other is the trivial state) were found at the interface of two BWAs with opposite propagation mismatches in the linear regime [20]. The JR state is well known for predicting the charge fractionalisation phenomenon which is fundamental in the fractional quantum Hall effect [21]. One of extraordinary features of the JR state is the topological nature of its zeroenergy solution which is considered to be a precursor to topological insulators [22]. Topological photonics can play a crucial role in the development of robust optical circuits [23]. In 2019, the JR states in interfaced BWAs were numerically demonstrated, as expected, to be also extremely robust under influence of strong disturbance of various kinds such as the turning on/off of the nonlinearity, the linear transverse potential, and the oblique incidence [24].

In the nonlinear regime, the exact profiles and the detunings of two localized states in interfaced BWAs have been found by using the so-called shooting method [25] both in the case of Kerr nonlinearity [26] and cubic-quintic nonlinearity [27]. The interaction between JR states and Dirac solitons in BWAs has been investigated in Ref. [28]. The interaction between two localized states in the nonlinear regime of Kerr type has been systematically analyzed in Ref. [29]. It has been shown in Ref. [29] that in the linear regime and the regime of Kerr nonlinearity two localized states of different types do not interact practically at all, thus they can be protected from each other in a reliable way even if they are located quite close to each other. Meanwhile, two localized states of the same type can couple efficiently to each other and show the nonlinear switching effect like in symmetric fiber couplers [29].

As mentioned above, the interaction between two localized states in the linear regime and in the regime of Kerr nonlinearity have been systematically investigated in Ref. [29]. The model with Kerr nonlinearity is the simplest one for investigating third-order nonlinear effects in optics. However, if the optical signals are intense enough one needs to take into account the fifth and even higher-order terms for nonlinearity. The resulting equation in that case is the well-known cubicquintic nonlinear Schrödinger equation (NLS) because it contains terms accounting for both the third and fifth powers of the signal amplitude. In fiber optics this cubic-quintic NLS for a single fiber has been well studied [30].

In this work we study the interaction between two relativistic quantum JR states in interfaced BWAs made of material with cubic-quintic nonlinearity. We show that in the low-intensity regime with cubic-quintic nonlinearity, like in the case with Kerr nonlinearity, two localized states of the same type can also couple efficiently, and if we increase further the input intensity of localized states then we also observe the nonlinear switching effect like in fiber couplers and the trapping effect of localized states. However, if the input intensity of localized states is high enough, then saturation effect of nonlinearity takes place and shows some interesting effects that the regime of Kerr nonlinearity cannot possess. We also confirm that two localized states of different types practically do not interact with each other at all in the linear and nonlinear regimes, both with Kerr and cubic-quintic nonlinearity.

The paper is organized as follows. In Sec. II, as a starting point, we give the theoretical background of two localized states solutions in interfaced BWAs in the linear regime which will be necessary further in this work. Then, in Sec. III, we investigate systematically the coupling and 
switching effects of two localized states of the same type in interfaced BWAs. Finally, in Sec. IV we summarize our results and finish with concluding remarks.

\section{GOVERNING EQUATIONS AND LINEAR SOLUTIONS OF JACKIW-REBBI STATES}

Light propagation in BWAs with cubic-quintic nonlinearity can be described, in the continuouswave regime, by the following dimensionless coupled-mode equations (CMEs) [12]:

$$
i \frac{d a_{n}}{d z}+\kappa\left[a_{n+1}+a_{n-1}\right]-(-1)^{n} \sigma a_{n}+\gamma\left(1-b_{s}\left|a_{n}\right|^{2}\right)\left|a_{n}\right|^{2} a_{n}=0,
$$

where $a_{n}$ is the electric field amplitude in the $n$th waveguide, $z$ is the longitudinal spatial coordinate, $2 \sigma$ and $\kappa$ are the propagation mismatch and the coupling coefficient between two adjacent waveguides of the array, respectively, $\gamma$ is the nonlinear coefficient of the cubic terms of waveguides, and $b_{s}$ is the saturation parameter governing the power level at which the nonlinearity begins to saturate. For many materials $b_{s}\left|a_{n}\right|^{2} \ll 1$ in most practical situations. However, this term may become relevant when the peak intensity approaches $1 \mathrm{GW} / \mathrm{cm}^{2}$ in the case of silica [31] (this peak intensity level can be easily achieved with pulses generated, for instance, from Ti-sapphire lasers and is commonly used in fiber optics [31]). Note that the cubic-quintic nonlinearity is also called competing nonlinearities and $b_{s}$ is always positive for most of optical materials such as semiconductor waveguides, semiconductor-doped glasses, and organic polymers [32]. The nonlinear terms in Eqs. (1) describe the competition between self-focusing occurring at low intensities due to the cubic term and self-defocusing taking over at higher intensities due to the quintic term [32]. For some materials such as single-crystal polydiacetylene paratoluene-sulfonate it has been experimentally demonstrated that the refractive nonlinear coefficient $n_{2}$ can change its sign from positive (which is responsible for self-focusing) to negative (which is responsible for self-defocusing) [33] when the optical intensity increases. It is also worth mentioning that the cubic-quintic nonlinearity is a special case of a more general kind of nonlinearity known as saturable nonlinearity which exists in many nonlinear media when the input intensity is much smaller than the so-called saturation intensity (see in Ref. [32, Eq. (7.4.1)]).

Now let us briefly re-introduce the linear localized solutions obtained at the interface of two BWAs with opposite propagation mismatches which have been found in Ref. [20]. These linear solutions will be used further for constructing the initial conditions to solve the beam propagation problem based on nonlinear Eqs. (1). Like in Ref. [20], now we set this interface at two central waveguides with $n=(-1,0)$ (see Fig. 1(a) in [20] for more details). For waveguides with $n<0$ we have $\sigma=\sigma_{1}$, where for $n \geq 0$ we have $\sigma=\sigma_{2}$. After setting $\Psi_{1}(n)=(-1)^{n} a_{2 n}$ and $\Psi_{2}(n)=$ $i(-1)^{n} a_{2 n-1}$, and following the standard approach in Ref. [11] we can introduce the continuous transverse coordinate $\xi \leftrightarrow n$ and the two-component spinor $\Psi(\xi, z)=\left(\Psi_{1}, \Psi_{2}\right)^{T}$ which satisfies the one-dimensional nonlinear Dirac equation:

$$
i \partial_{z} \Psi=-\mathrm{i} \kappa \hat{\sigma}_{x} \partial_{\xi} \Psi+\sigma \hat{\sigma}_{z} \Psi-\gamma G+\gamma b_{s} F,
$$

where the cubic nonlinearity is taken into account via the term $G \equiv\left(\left|\Psi_{1}\right|^{2} \Psi_{1},\left|\Psi_{2}\right|^{2} \Psi_{2}\right)^{T}$; the quintic nonlinearity is taken into account via the term $F \equiv\left(\left|\Psi_{1}\right|^{4} \Psi_{1},\left|\Psi_{2}\right|^{4} \Psi_{2}\right)^{T} ; \hat{\sigma}_{x}$ and $\hat{\sigma}_{z}$ are the usual Pauli matrices. In quantum field theory the parameter $\sigma$ in the Dirac equation is often 
called the mass of the Dirac field (or Dirac mass). In the case of just Kerr nonlinearity the resulting equation will be simplified as Eq. (7) in Ref. [12] without the quintic term.

If $\sigma_{1}<0$ and $\sigma_{2}>0$ we get the following localized profile as an exact JR solution of Eq. (2) in the linear case [20]:

$$
\Psi(\xi)=\sqrt{\frac{\left|\sigma_{1} \sigma_{2}\right|}{\kappa\left(\left|\sigma_{1}\right|+\left|\sigma_{2}\right|\right)}}\left(\begin{array}{c}
1 \\
i
\end{array}\right) e^{-|\sigma(\xi) \xi| / \kappa} .
$$

If $\left|\sigma_{1}\right|=\left|\sigma_{2}\right|=\sigma_{0}$ one can get exact localized solutions for the discrete Eq. (1) without nonlinearity $(\gamma=0)$ for the following two cases [20,27,29]:

If $-\sigma_{1}=\sigma_{2}=\sigma_{0}>0$, one gets the following discrete JR state $[20,27,29]$ :

$$
a_{n}=b_{n} e^{i \delta_{1} z}
$$

where the detuning $\delta_{1} \equiv \kappa-\sqrt{\sigma_{0}^{2}+\kappa^{2}}, b_{n}$ is real and independent of the variable $z, b_{2 n-1}=b_{2 n}$, if $n \geq 0$ one has the following relationship: $b_{2 n} / b_{2 n+1}=\alpha \equiv-\left[\sigma_{0} / \kappa+\sqrt{1+\sigma_{0}^{2} / \kappa^{2}}\right]$, whereas for $n<0$ one has: $b_{2 n} / b_{2 n+1}=\alpha$. Note that the interface supporting this trivial state has two adjacent waveguides with $(-1)^{n} \sigma$ which must be positive (see also Fig. 1(a) for more details). However, if $\sigma_{1}=-\sigma_{2}=\sigma_{0}>0$, one has the following localized trivial state [20,27, 29]:

$$
a_{n}=b_{n} e^{i \delta_{2} z},
$$

where the detuning $\delta_{2} \equiv \kappa+\sqrt{\sigma_{0}^{2}+\kappa^{2}}, b_{n}$ is again real and independent of the variable $z, b_{2 n-1}=$ $b_{2 n}$, if $n \geq 0$ one has $b_{2 n} / b_{2 n+1}=-\alpha$, whereas for $n<0$ one has: $b_{2 n+1} / b_{2 n}=-\alpha$. Note that the interface supporting this trivial state has two adjacent waveguides with $(-1)^{n} \sigma$ which must be negative.

Because the solutions in the form of Eqs. (3) - (5) are obtained in the linear case, obviously, one will get other linear solutions by multiplying them by an arbitrary number. In other words, the peak amplitudes of linear solutions can be chosen arbitrarily. Even though these solutions are obtained in the linear case, as pointed out in Ref. [20], one can use them to construct the initial conditions for getting the robust nonlinear discrete JR states which can be self-adjusted and established further during propagation. If the exact localized nonlinear localized states solutions are needed at the very beginning, then one can use the shooting method to numerically find them as shown in Ref. [26]. In the rest of this work, we will numerically solve Eqs. (1) to investigate the interaction between two discrete JR states in interfaced BWAs. As initial conditions for this task we will use beams in the form of Eq. (3) multiplied by a certain factor $f$ which will be specified later in each case. In the nonlinear case, if the system consists of just two interfaced BWAs, then after some propagation distance, the nonlinear JR states will adjust their profiles and get stable ones, provided that the factor $f$ is not too different from unity [20].

To estimate real physical parameters of the calculated DS solitons below we use typical parameters in waveguide arrays made of AlGaAs [34], where the coupling coefficient and nonlinear coefficient in physical units are $K=1240 \mathrm{~m}^{-1}$ and $\Gamma=6.5 \mathrm{~m}^{-1} \mathrm{~W}^{-1}$, respectively. In this case, the power scale will be $P_{0}=K / \Gamma=190.8 \mathrm{~W}$ and the length scale in the propagation direction will be $z_{0}=1 / K=0.8 \mathrm{~mm}$. 


\section{COUPLING AND SWITCHING EFFECTS OF TWO DISCRETE JACKIW-REBBI STATES}

Now it is ready for us to deal with the main targets of this work, i.e., to investigate the interaction of two discrete JR states in the regime of the cubic-quintic nonlinearity. In this work we will focus on the interaction of discrete JR states, because the interaction of trivial states also possesses the same qualitative features. Fig. 1(a) plots the array of $(-1)^{n} \sigma$ where the interface at the two waveguides with $n=(-5,-4)$ and the interface at the two waveguides with $n=(4,5)$ can both generate discrete JR states. The system consists of three BWAs: the first one covers all waveguides with $n \leq-5$ and has the propagation mismatch parameter $\sigma_{1}$, the second one covers all waveguides with $-4 \leq n \leq 4$ and has the propagation mismatch parameter $\sigma_{2}$, and the third one covers all waveguides with $n \geq 5$ and has the propagation mismatch parameter $\sigma_{3}$. Other parameters used for obtaining results in Fig. 1 are as follows: the coupling coefficient $\kappa=1$, the nonlinear coefficient for the cubic term $\gamma=1$, and the saturation parameter $b_{s}=0.4$. In Fig. 1(b) we launch a discrete JR state with $f=0.1$ at the interface at the two waveguides with $n=(4,5)$. Because the input beam used in Fig. 1(b) is so weak with $f=0.1$, so even though the full cubicquintic model based on Eqs. (1) is used for simulation, we practically operate in the linear regime in Fig. 1(b) and obtain almost the same result as shown in Fig. 5(b) in Ref. [29] with just cubic nonlinearity term and $f=0.1$ as well. What shown in Fig. 1(b) is a typical coupling between two linear discrete JR states with the coupling length $L=106$ where all the energy of the JR state at the interface with $n=(4,5)$ is transferred to the JR state at the interface with $n=(-5,-4)$. At the propagation distance $z=2 L$ all the energy is again completely transferred back to the JR state at the interface with $n=(4,5)$. This kind of energy transfer (or coupling) between the two JR states takes place periodically. As pointed out in Ref. [29], this linear coupling between two discrete JR states is completely similar to the well-known coupling between signals in two cores of a symmetric fiber coupler [3]. The physics behind the coupling between two discrete JR states is due to the phase matching condition between these two linear discrete JR states [29]. Indeed, as clearly shown in the analytical solutions in the forms of Eqs. (4), in the linear (or low-power) regime two discrete JR states always have the same phase during propagation, providing that $\left|\sigma_{1}\right|=\left|\sigma_{2}\right|=\left|\sigma_{3}\right|$ and $\kappa$ is constant for the whole system. Therefore, the phase matching during propagation between these discrete JR states in the linear regime is always satisfied which results in the efficient coupling between them. In Fig. 1(c) the factor $f$ is increased up to the value $f$ $=0.5$. Now the beam propagation pattern is again periodic during propagation, but only a small amount of total energy is periodically transferred to the interface with $n=(-5,-4)$, whereas the main part of the total energy is trapped at the interface with $n=(4,5)$. This situation is totally different from the scenarios in Fig. 1(b) where all energy is periodically transferred between the two interfaces. This behavior of the nonlinear coupling between two discrete JR states is again completely similar to the nonlinear coupling scenario in symmetric fiber couplers when the input power $P_{0}$ is larger than the critical power $P_{c}$ (see Ref. [3, p. 63]). As compared to the case shown in Fig. 5(d) in Ref. [29] with the same factor $f=0.5$, but the nonlinearity is of the pure Kerr type (i.e., $b_{s}=0$ in Fig. 5(d) in Ref. [29] instead of $b_{s}=0.4$ in Fig. 1(c) of this current work), one can see that all the qualitative features in these two figures are the same with the only exception that the period in Fig. 5(d) in Ref. [29] is a little bit shorter than the one in Fig. 1(c) of this current work. If we increase further the factor $f$ up to the value $f=1$, then our simulations (not included 

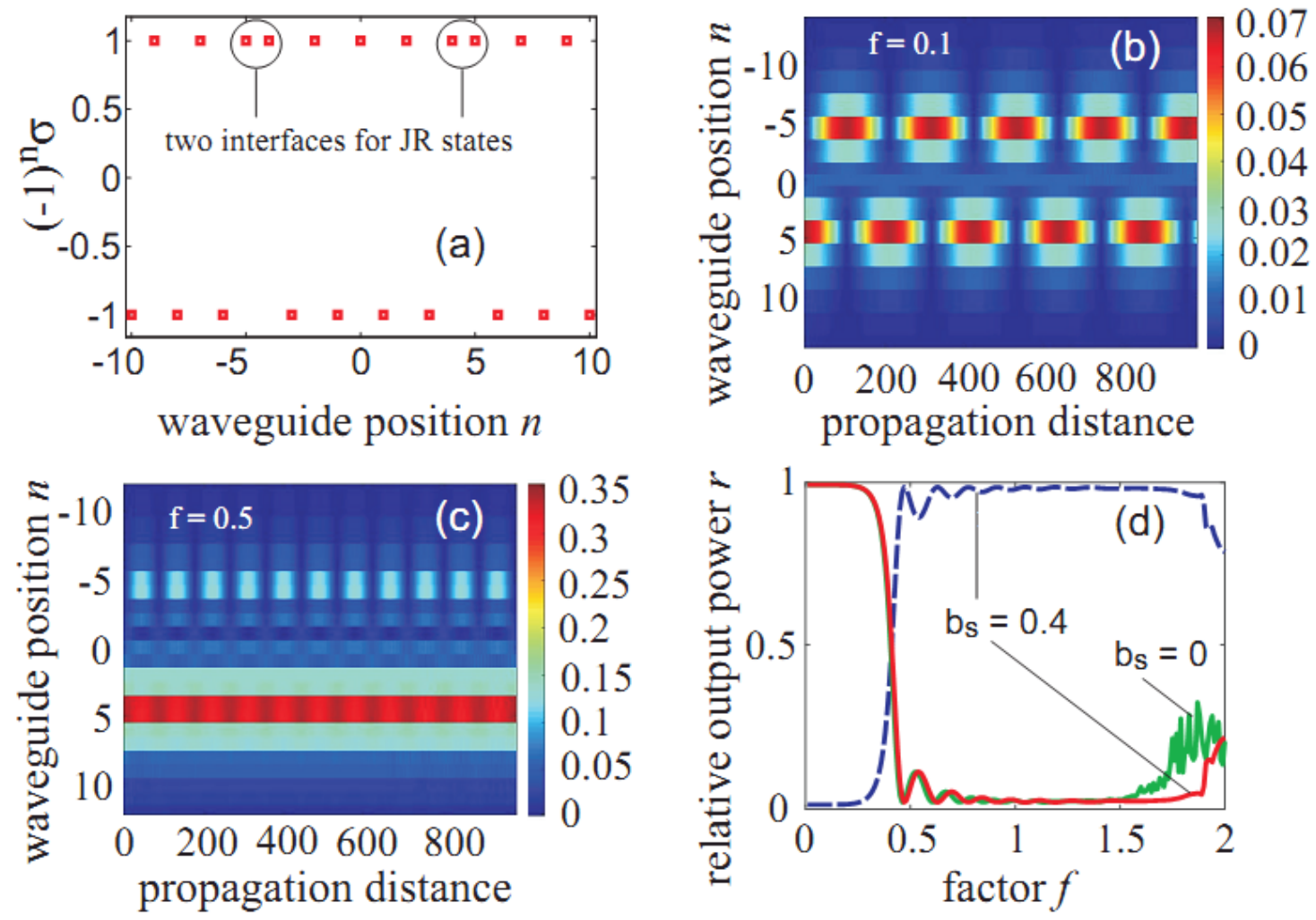

Fig. 1. (Color online) Coupling of two discrete JR states. (a) The array of $(-1)^{n} \sigma$ as a function of the waveguide position $n$. (b,c) Coupling of two discrete JR states when $f=$ 0.1 and 0.5 , respectively. (d) The switching character of two discrete JR states when the longitudinal length of waveguide arrays is equal to the linear coupling length $L=106$. Parameters: $-\sigma_{1}=\sigma_{2}=-\sigma_{3}=1 ; \kappa=1 ; \gamma=1 ; b_{s}=0.4$.

here) show that the input JR state is practically trapped at the interface with $n=(4,5)$ where it was launched into as already demonstrated in Fig. 5(e) in Ref. [29] for Kerr nonlinearity. This case is again completely similar to the trapping effect in symmetric fiber couplers when $P_{0} \gg P_{c}$ [3]. Therefore, like the situation in fiber couplers and the interaction between two discrete JR states with Kerr nonlinearity, we have shown that in the regime of cubic-quintic nonlinearity a discrete JR state can be switched from one interface to the other, or trapped at one interface, depending on its input power, provided that these two interfaces can support the discrete JR states.

In Fig. 1(d) we plot the relative output power of the two JR states (with respect to the total initial input power) at the propagation distance equal to the coupling length $L=106$ as a function of the factor $f$ where the full model with the cubic-quintic nonlinearity based on Eqs. (1) is used. The solid red (dashed blue) curve in Fig. 1(d) represents the relative output power of the JR state by summing up $\left|a_{n}\right|^{2}$ for all $n<0(n>0)$ with the saturation parameter $b_{s}=0.4$. For comparison in Fig. 1(d) we also plot the green solid curve with label $b_{s}=0$ which represents the relative output 
power of the JR state with all $n<0$ in the case of Kerr nonlinearity. The latter curve has been already shown in Fig. 5(f) in Ref. [29] (see the solid red curve therein). Now let us focus on the similarities of the solid red curve and the solid green curve in Fig. 1(d). One can see that in the low-power regime (when $f<0.2$ ) all the energy of the initial JR state with $n>0$ is also completely transferred to the JR state with $n<0$. As a result, the solid red curve is close to unity, whereas the dashed blue curve is close to zero. However, in the nonlinear regime (when $0.8<f<1.8$ ) all the energy of the input JR state is also just trapped at the same interface, and almost nothing is transferred to the other JR state. By changing the factor $f$ around 0.4 one also can obtain the switching effect of the JR states between two interfaces. So, with the model for both Kerr and cubic-quintic nonlinearity, the features of the linear coupling and nonlinear switching between two discrete JR states are quite similar to the ones between optical signals in symmetric fiber couplers (see Ref. [3, Fig. 2.3]). It is worth mentioning that in fiber couplers the coupling and switching happen just between two cores, whereas for JR states they happen between two interfaces (each interface consists of two and nearby waveguides). Our simulations also confirm that the coupling and switching effects of two trivial states (not shown here) have exactly the same features as the ones of two discrete JR states illustrated in Fig.1 as mentioned in Ref. [29], the physical reason behind the nonlinear switching between two discrete JR states is similar to the reason in the case of symmetric fiber couplers [3]. The difference in the mode propagation constants due to nonlinearity-based self-phase modulation (SPM) introduces a relative phase shift between the two JR states and hinders complete power transfer between them. At sufficiently high input powers, the phase difference due to SPM becomes large enough that the input JR state remains trapped in the same interface into which it was initially launched. Note that under ideal conditions, the response time of the switching effect is limited by the response time of the cubic-quintic nonlinearity which is almost instantaneous. For silica, this response time is less than $10 \mathrm{fs}$ [31].

Now let us analyze the differences between the solid red curve and the solid green curve in Fig. 1(d). First, when the factor $f<1.5$ the red solid curve is slightly shifted to the right as compared to the solid green curve. This little shift of the red solid curve can be better seen by enlarging Fig. 1(d). Second, when $f$ is too large (close to 2) one can see that both two curves rise up again. However, the mechanism for this rising is totally different for these two curves. Regarding the green solid curve representing the case with Kerr nonlinearity, when $f$ is too large, the input condition is too different from the nonlinear localized JR states investigated in Ref. [27]. So, after launching these intense beams into the system, our simulations (not shown here) reveal that a significant amount of the input energy is immediately radiated in a chaotic manner to both two sides of the system. That is the reason why the whole input beam is not trapped at the input interface, but some energy is distributed to the other side of the system which leads to the rising of the green solid curve. On the contrary, the rising of the red curve around $f=2$ in Fig. 1(d) happens in an orderly manner because of the saturation effect which makes the nonlinear terms less important in that region, thus forces the system operate in a regime close to the transition regime around $f=0.4$ of the same curve. This mechanism will be clarified in more details in the rest of this work.

In Figs. 2(a,c,e) we investigate the dependence of the relative output power $r$ as a function of the multiplication factor $f$ for three values of the saturation parameter $b_{s}=0.6,0.8$ and 1.0, respectively. In order to better understand the influence of the nonlinear terms in Eqs. (1), let us 

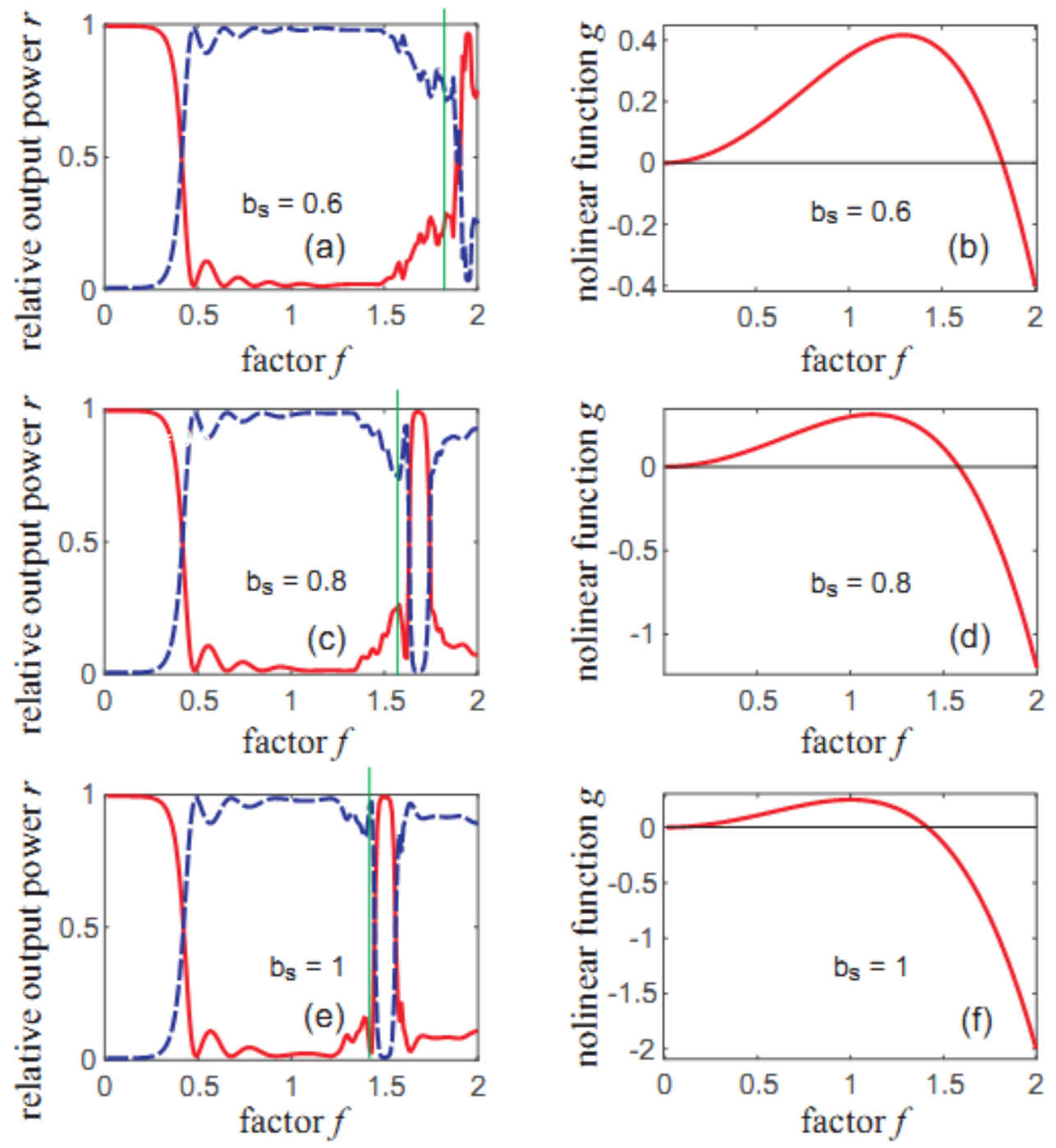

Fig. 2. (Color online) (a,c,e) The switching character of two discrete JR states when the longitudinal length of waveguide arrays is equal to the linear coupling length $L=106$ for the saturation parameter $b_{s}=0.6,0.8$ and 1.0 , respectively. (b,d,f) The nonlinear function $g$ as a function of the factor $f$ for the saturation parameter $b_{s}=0.6,0.8$ and 1.0, respectively. The position where $g=0$ is indicated by the vertical green solid lines in (a,c,e), respectively. Parameters: $-\sigma_{1}=\sigma_{2}=-\sigma_{3}=1 ; \kappa=1 ; \gamma=1$.

define the nonlinear function $g(f)$ as follows:

$$
g(f)=\left[1-b_{s}(p f)^{2}\right](p f)^{2},
$$

where the parameter $p=\sqrt{\left|\sigma_{1} \sigma_{2}\right| /\left[\kappa\left(\left|\sigma_{1}\right|+\left|\sigma_{2}\right|\right)\right]}$, thus $p f$ represents the peak amplitude of the interface with $n=(4,5)$. In Figs. 2(b,d,f) we plot the nonlinear function $g$ as a function 
of the multiplication factor $\mathrm{f}$ for three values of the saturation parameter $b_{s}=0.6,0.8$ and 1.0 , respectively. As clearly seen in Figs. 2(b,d,f), when the factor $f$ is small, the nonlinear function $g$ and the factor $f$ increase at the same time. This is understandable because small values of $f$ mean that the peak amplitude of the input JR state is also weak, and thus the cubic term of the nonlinearity in Eqs. (1) prevails over the quintic term. However, when $f$ is increased further, the quintic term now can compete with the cubic term which leads to the saturation effect of the nonlinearity. As a result, the nonlinear function $g$ cannot increase further, but gets its maximum value, and decreases for large values of $f$. At the value $f=1 /\left(p \sqrt{b_{s}}\right)$ the nonlinear function $g$ will be nullified. These positions of $f$ are indicated in Figs. 2(a,c,e) by the vertical green solid lines. If the factor $f$ is increased further, then the nonlinear function $g$ will become negative which means that the nonlinearity now is self-defocusing for the peak amplitude at the input. As already mentioned in the Introduction, it has been experimentally demonstrated that some materials can switch the nonlinearity from self-focusing to self-defocusing when the input signal is intense enough [33]. So, now it is clear why the relative output power $r$ can rise up to unity again in Figs. 2(a,c,e) when $f$ is around the values which can nullify the nonlinear function $g$ (i.e., around the vertical green solid lines in Figs. 2(a,c,e)). This is because around these high values of $f$ the system again operates in the quasi-linear regime which is similar to the truly linear regime for small values of $f$, and thus at the coupling length $L=106$ almost all energy of the input JR state from the interface with $n=(4,5)$ can be transferred to the JR state at the interface with $n=(-5,-4)$, like what happens with small values of $f$ in the low-intensity regime in Fig. 1(b).
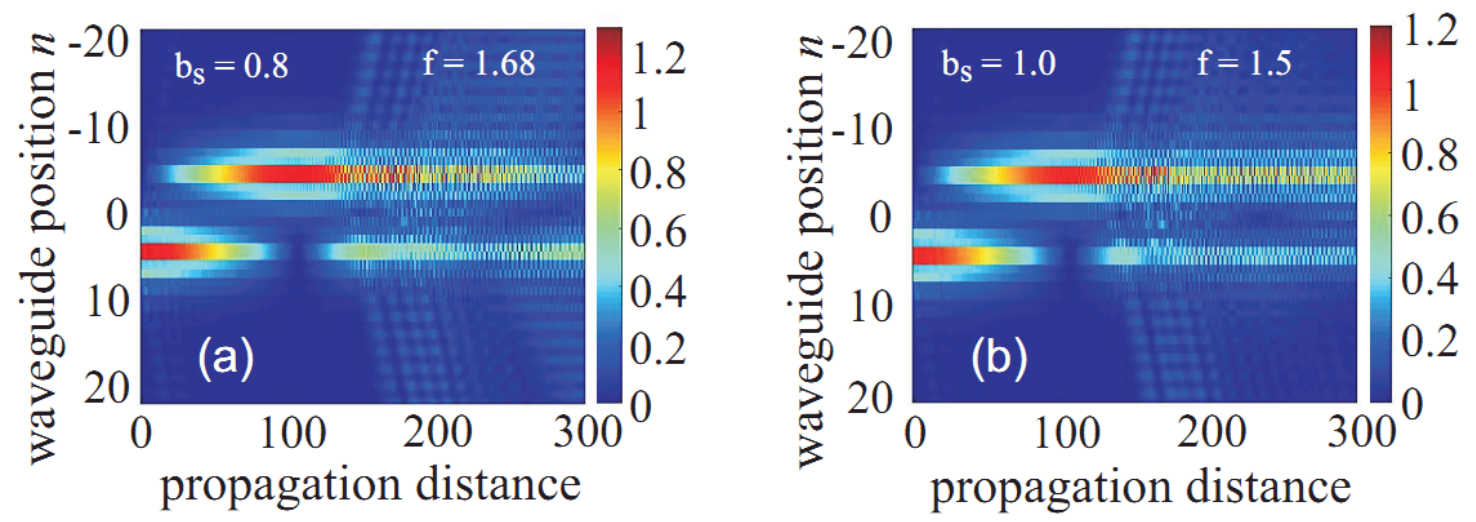

Fig. 3. (Color online) (a) Coupling of two discrete JR states when $b_{s}=0.8$ and $f=1.68$. (b) Coupling of two discrete JR states when $b_{s}=1.0$ and $f=1.5$. Parameters: $-\sigma_{1}=$ $\sigma_{2}=-\sigma_{3}=1 ; \kappa=1 ; \gamma=1$.

The possibility that the relative output power $r$ can rise up again to unity for a certain large value of $f$ is demonstrated in Figs. 3(a,b) where the propagation of the input JR beams is illustrated for $b_{s}=0.8$ with the multiplication factor $f=1.68$ in Fig. 3(a), and for $b_{s}=1.0$ with the multiplication factor $f=1.5$ in Fig. 3(b). Both these input JR states are launched into the system at the interface with $n=(4,5)$. As expected, at the coupling length $L=106$, all the energy of these JR states is transferred to the JR states at the interface with $n=(-5,-4)$, like what happens in 
the linear regime. However, in the truly linear regime, this energy transfer between two interfaces takes place periodically as clearly shown in Fig. 1(b), whereas this periodicity is not present in the quasi-linear regime in Fig. 3. This is because in the truly near regime, or in the regime with small values of $f$ as shown in Fig. 1(b), one can totally neglect the nonlinear terms from Eqs. (1), and the system is indeed a linear one. However, for high values of $f$ as in Fig. 3 one cannot do that, because the nonlinear function $g$ is only calculated for the peak amplitude of the beam at the input, but not for all components $a_{n}$ of the beams. Therefore, even though at some values of $f$ the nonlinear function $g$ defined in Eq. (6) can be nullified for the peak amplitude at the input (i.e., for $a_{4}$ and $a_{5}$ at the input), but the nonlinear terms for $a_{3}, a_{6}$ and other components will significantly differ from zero at the input. That is the reason we use the term quasi-linear for the high-intensity regime close to the vertical green solid curves in Figs. 2(a,c,e).

It is worth mentioning that in the regime of Kerr nonlinearity, because $b_{s}=0$, therefore the nonlinear function $g$ is a parabolic function of the factor $f$. Thus, the nonlinear function $g$ quickly increases without any saturation effect when the factor $f$ is increased. So in the high-intensity regime, the dynamics of these two cases (Kerr nonlinearity and cubic-quintic nonlinearity) will be totally different, and one cannot observe the switching scenarios as in Figs. 2(a,c,e) at large values of $f$ (around the vertical green lines) for Kerr nonlinearity.

Even though in this work we only show explicitly the interaction between two discrete JR states in interfaced BWAs with cubic-quintic nonlinearity, our simulations (not included here) confirm that the interaction between two trivial states also possesses exactly the same qualitative features, and that two localized states of different types practically do not interact at all in the regime of cubic-quintic nonlinearity as what happens in the regime with Kerr nonlinearity.

\section{CONCLUSIONS}

In conclusion, we have shown that for both Kerr nonlinearity and cubic-quintic nonlinearity the coupling and switching effects between two discrete JR states in interfaced BWAs are quite similar to what happens in symmetric fiber couplers: for low input intensities one can observe the strong coupling between these two JR states, for higher intensities one can observe the switching effect, whereas for even higher intensities the trapping effect of the JR states will take place. However, if the JR state input intensity is increased up further, then the saturation effect of the cubic-quintic nonlinearity will be important, which can lead to the strong coupling between two discrete JR states again. This coupling in the quasi-linear regime for high input intensities with cubic-quintic nonlinearity does not take place periodically during propagation, unlike coupling at low intensities. Regarding two localized states of different types in interfaced BWAs, like in the case of the linear regime and Kerr nonlinearity, they do not interact at all in the regime of cubicquintic nonlinearity. This kind of interaction between two localized states can potentially be useful for optical switching, coupling and robust guiding in photonic integrated circuits.

\section{ACKNOWLEDGMENT}

This research is funded by Vietnam National Foundation for Science and Technology Development (NAFOSTED) under grant number 103.03-2019.03. 


\section{REFERENCES}

[1] D. N. Christodoulides, F. Lederer, and Y. Silberberg, Nature 424 (2003) 817.

[2] D. N. Christodoulides and R. I. Joseph, Opt. Lett. 13 (1988) 794.

[3] G. P. Agrawal, Applications of Nonlinear Fiber Optics, 2nd. (Academic Press, New York, 2008).

[4] A. L. Jones, J. Opt. Soc. Am. 55 (1965) 261.

[5] Tr. X. Tran and F. Biancalana, Phys. Rev. Lett. 110 (2013) 113903.

[6] M. Ghulinyan, C. J. Oton, Z. Gaburro, L. Pavesi, C. Toninelli, and D. S. Wiersma, Phys. Rev. Lett. 94 (2005) 127401.

[7] T. Pertsch, P. Dannberg, W. Elein, A. Bräuer, and F. Lederer, Phys. Rev. Lett. 83 (1999) 4752.

[8] S. Longhi, Phys. Rev. B 81 (2010) 075102.

[9] F. Dreisow, R. Keil, A. Tünnermann, S. Nolte, S. Longhi, and A. Szameit, Europhys. Lett. 97 (2012) 10008.

[10] Q. Nguyen-The and Tr. X. Tran, J. Opt. Soc. Am. B 37 (2020) 1911.

[11] F. Dreisow, M. Heinrich, R. Keil, A. Tünnermann, S. Nolte, S. Longhi, and A. Szameit, Phys. Rev. Lett. 105 (2010) 143902.

[12] Tr. X. Tran, S. Longhi, and F. Biancalana, Ann. Phys. 340 (2014) 179.

[13] Tr. X. Tran, X. N. Nguyen, and D. C. Duong, J. Opt. Soc. Am. B 31 (2014) 1132.

[14] Tr. X. Tran, X. N. Nguyen, and F. Biancalana, Phys. Rev. A 91 (2015) 023814.

[15] Tr. X. Tran and D. C. Duong, Ann. Phys. 361 (2015) 501.

[16] Tr. X. Tran., H. M. Nguyen, and H. D. Phung, Commun. in Phys. 27 (2017) 205.

[17] Tr. X. Tran and D. C. Duong, Chaos 28 (2018) 013112.

[18] Tr. X. Tran, J. Opt. Soc. Am. B 36 (2019) 2001.

[19] R. Jackiw and C. Rebbi, Phys. Rev. D 13 (1976) 3398.

[20] Tr. X. Tran and F. Biancalana, Phys. Rev. A 96 (2017) 013831.

[21] R. B. Laughlin, Rev. Mod. Phys. 71 (1999) 863.

[22] M. Z. Hasan and C. L. Kane, Rev. Mod. Phys. 82 (2010) 3045.

[23] M. C. Rechtsman, J. M. Zeuner, Y. Plotnik, Y. Lumer, D. Podolsky, F. Dreisow, S. Nolte, M. Segev, and A. Szameit, Nature 496 (2013) 196.

[24] Tr. X. Tran, J. Opt. Soc. Am. B 36 (2019) 2559.

[25] N. N. Rosanov and Tr. X. Tran, Chaos 17 (2007) 037114.

[26] Tr. X. Tran, H. M. Nguyen, and D. C. Duong, Phys. Rev. A 100 (2019) 053849.

[27] Tr. X. Tran, Chaos 30 (2020) 063134.

[28] Tr. X. Tran, D. C. Duong, and F. Biancalana, J. Lightwave Technol. 35 (2017) 5092.

[29] Tr. X. Tran, Phys. Rev. A 101 (2020) 063826.

[30] N. Akhmediev and A. Ankiewicz, Eds., Dissipative Soli-tons (Springer, New York, 2005).

[31] G. P. Agrawal, Nonlinear Fiber Optics, 5th ed. (Academic Press, New York, 2013).

[32] Y. S. Kivshar and G. P. Agrawal, Optical Solitons: from Fiber to Photonic Crystals, 5th ed. (Academic, New York, 2003).

[33] B. Lawrence, W. E. Torruellas, M. Cha, M. L. Sundheimer, and G. I. Stegeman, Phys. Rev. Lett. 73 (1994) 597.

[34] R. Morandotti, U. Peschel, J. S. Aitchison, H. S. Eisenberg, and Y. Silberberg, Phys. Rev. Lett. 83 (1999) 4756. 